Article

\title{
Fractional Differential Generalization of the Single Particle Model of a Lithium-Ion Cell
}

\author{
Renat T. Sibatov ${ }^{1, *,+}\left(\mathbb{D}\right.$, Vyacheslav V. Svetukhin ${ }^{2, \dagger}$, Evgeny P. Kitsyuk ${ }^{2,+}$ and \\ Alexander A. Pavlov ${ }^{3,+}$ \\ 1 Laboratory of Diffusion Processes, Ulyanovsk State University, Ulyanovsk 432017, Russia \\ 2 Scientific Manufacturing Complex "Technological Centre", Moscow 124498, Russia; \\ svetukhin@mail.ru (V.V.S.); kitsyuk.e@gmail.com (E.P.K.) \\ 3 Institute of Nanotechnology of Microelectronics of the Russian Academy of Sciences (INME RAS), \\ Moscow 119991, Russia; alexander.a.pavlov@gmail.com \\ * Correspondence: ren_sib@bk.ru; Tel.: +7-842-267-5054 \\ + These authors contributed equally to this work.
}

Received: 7 May 2019; Accepted: 7 June 2019; Published: 9 June 2019

\begin{abstract}
The effect of anomalous diffusion of lithium on the discharge curves and impedance spectra of lithium-ion batteries (LIB) is studied within the fractional differential generalization of the single-particle model. The distribution of lithium ions in electrolyte and electrode particles is expressed through the Mittag-Leffler function and the Lévy stable density. Using the new model, we generalize the equivalent circuit of LIB. The slope of the low-frequency rectilinear part of the Nyquist diagram does not always unambiguously determine the subdiffusion index and can be either larger or smaller than the slope corresponding to normal diffusion. The new aspect of capacity degradation related to a change in the type of ion diffusion in LIB components is discussed.
\end{abstract}

Keywords: lithium-ion battery; single-particle model; fractional derivative; anomalous diffusion; impedance; degradation; equivalent circuit; impedance

\section{Introduction}

Mathematical modeling of lithium-ion batteries (LIB) is necessary both to optimize the design and operation modes and to test the state of working devices: to assess state-of-health, state-of-charge, and predict performance [1,2]. The charging and discharging of LIBs are followed by complex processes including reaction at the electrode/electrolyte interface, lithium transport in solid electrode particles, the formation of an electrical double layer, the growth of a solid-electrolyte interface (SEI), phase transformations, and redistribution of stresses during lithiation and delithiation, among others.

Recently, LIB models based on fractional-order impedances have been proposed [3-5]. These models are justified by the anomalous diffusion of ions in a disordered medium of a percolative type. In [6], the authors used the phenomenological model accounting for anomalous ion diffusion in a $\mathrm{LiCoO}_{2}$ cathode to describe the low-frequency part of the Nyquist diagram that has a steeper slope than predicted by normal diffusion. Using the subdiffusion generalization [7] of the electrochemical impedance, the authors of [6] showed that the anomalous-diffusion model describes the impedance of $\mathrm{LiCoO}_{2} \mid \mathrm{C}$ cells well at normal operating potentials and temperatures. They noted that the contribution of anomalous diffusion decreases for higher temperatures. Some experimental studies (see, e.g., [8-10]) using nuclear magnetic resonance (NMR) clearly showed that the diffusion of ions and molecular complexes in some media is characterized by features typical of anomalous diffusion.

The mathematical basis of anomalous self-similar diffusion is formed by equations with fractional-order derivatives [11-13]. Sabatier et al. [14,15] argued that fractional differentiation helps to 
describe some components of a model with a smaller number of parameters related to electrochemical quantities. Such fractional models with a reduced number of fitting parameters can be used for the development of state-of-charge (SOC) and state-of-health ( $\mathrm{SOH}$ ) estimators. In [14,15], fractional models were used as simplifications of an integer-order electrochemical model, and the authors did not consider the anomalous diffusive behavior of ion transport in electrolyte and electrode particles.

Some solid electrolytes used in LIB, for example, compound $\mathrm{Li}_{7} \mathrm{La}_{3} \mathrm{Zr}_{2} \mathrm{O}_{12}$ (LLZO), have a polycrystalline structure with grain boundaries (GBs). As is known, GBs can accelerate or suppress the diffusion of atoms and ions in polycrystalline materials (see [16]). Many approaches to the description of grain boundary diffusion are based on the classical model proposed by Fisher in 1951. They use a system of normal-diffusion equations in a heterogeneous medium. A direct relationship between the fractional differential model of anomalous diffusion and the Fisher model and its modifications without additional assumptions was derived in [17] (see also [18]).

Many models of ion transport in a porous electrode are based on the effective medium approximation, where two parameters are introduced: the volume fraction of space available for transfer and the tortuosity of flow paths. With the use of fractal geometry and fractional calculus, Huang [19] proposed a new model to describe the mass transfer and reactions in LIB electrodes having a fragmented fractal structure.

Despite the observed evidence of anomalous diffusion and the success of fractional circuit models, there are no microscopic models that jointly describe anomalous diffusion and percolation of lithium ions in porous electrodes, SEI, and electrode particles. In this work, we present an attempt to make such a generalization using the fractional modification of the single-particle (SP) model.

\section{Fractional Differential Diffusion Equation}

Anomalous diffusion is often characterized by the power law expansion of the diffusion packet, $\Delta(t) \propto t^{\alpha / 2}$. The classical diffusion $(\alpha=1)$ implies Brownian motion of particles and the Fickian relation between current and concentration. When $0<\alpha<1$, the diffusion packet width increases with time more slowly than in the normal case (subdiffusion). If $\alpha>1$, it increases more quickly (superdiffusion). The mathematical basis of anomalous self-similar diffusion is formed by equations with fractional-order derivatives [11-13]. It should be noted, however, that linear growth of the diffusion packet width does not always correspond to ordinary diffusion (see, e.g., [20]) and can be observed for processes with "diffusing diffusivities" characterized by non-Gaussian distributions.

The dispersive diffusion (when delay is associated with localization times) can be described by the following integro-differential equation (see the reviews $[11,13,21]$ ):

$$
\frac{\partial c(\mathbf{r}, t)}{\partial t}=\int_{0}^{t} Q(t-\tau) \nabla^{2} c(\mathbf{r}, t) d \tau
$$

where $Q(t)$ is a memory kernel and $c(\mathbf{r}, t)$ is a particle concentration. In the subdiffusive regime, the requirement of self-similarity of the Green's functions leads to the fractional-order diffusion equation [11]:

$$
\frac{\partial c(x, t)}{\partial t}=K \nabla^{2}{ }_{0} \mathrm{D}_{t}^{1-\alpha} c(x, t) .
$$

Here:

$$
{ }_{0} \mathrm{D}_{t}^{v} c(x, t)=\frac{1}{\Gamma(1-v)} \frac{\partial}{\partial t} \int_{0}^{t} \frac{c(x, \tau)}{(t-\tau)^{v}} d \tau, \quad 0<v \leq 1
$$

is the fractional Riemann-Liouville derivative of order $v$ [22], $\Gamma(v)$ is the gamma function, and $K$ is a subdiffusion coefficient. Fundamental solutions to this equation can be found in [11]. Fractional differential Equation (2) can be obtained as the asymptotic equation describing the continuous time random walk (CTRW) [13] with "heavy-tailed" distributions of random localization times $\tau$ : $P\{\tau>t\} \propto t^{-\alpha}(0<\alpha<1)$. 
The power law distribution of localization times can be obtained, for example, in the random activation energy model. Suppose that ion hopping frequency is defined by a semiclassical expression $W=A e^{-\Delta E / k T}$, and the conditional distribution of waiting time corresponding to a given activation energy $\Delta E=\varepsilon$ is exponential: $\mathrm{P}\{T>t \mid \varepsilon\}=e^{-W(\varepsilon) t}$. If the activation energy is a random variable with the following distribution density $p(\varepsilon)=\varepsilon_{0}^{-1} e^{-\varepsilon / \varepsilon_{0}}$, then averaging over activation energies leads to the waiting time distribution of the power law type:

$$
\mathrm{P}\{T>t\}=\int_{0}^{\infty} \mathrm{P}\{T>t \mid \varepsilon\} p(\varepsilon) d \varepsilon=\int_{0}^{\infty} \exp \left[-\left(A e^{-\varepsilon / k T}\right) t\right] d\left(e^{-\varepsilon / \varepsilon_{0}}\right)=\alpha \Gamma(\alpha)(A t)^{-\alpha} .
$$

with $\alpha=k T / \varepsilon_{0}$. Here, $\varepsilon_{0}$ is a parameter characterizing the dispersion of activation energy. Under condition $k T<\varepsilon_{0}$, ion transport is subdiffusive.

Equation (2) can be justified within other transport models (see, e.g., [23]). In electrodes based on porous and nanostructured materials, there are many alternative transport paths, and ion transport is often percolative. To study diffusion on percolation clusters, the comb model [24] is often used. In the simplest version [24,25], the order of the fractional derivative in Equation (2) is equal to 1/2. In more complex versions, for example, in the hierarchical comb model [26] or in the model with random finger lengths [27], other values of the subdiffusion parameter can be obtained.

\section{Subdiffusion Model of the Lithium-Ion Cell}

To assess the effect of anomalous diffusion on the discharge characteristics of LIB and impedance spectra, we consider the subdiffusive generalization of the single-particle (SP) model, which is a simplification of the pseudo-two-dimensional (P2D) model (Figure 1). The original P2D-model was developed in $[1,28,29]$. The segment $[0, L]$ on the $x$ axis is considered to be divided into three sections corresponding to two electrodes and a separator. Newman and Tobias [30] showed that the current distribution in the porous electrode due to the electrochemical reaction is fairly uniform. The assumption about homogeneous current across the electrode thickness is a key point of the model. In the standard SP model, electrodes are modeled as two spherical particles, and the variations in electrolyte concentration and in potential are usually ignored. Although, there are generalizations (see, e.g., [31,32]), where these variations are taken into account. Here, we also consider changes in concentration and potential along the $x$-axis. Spread in particle sizes can be taken into account within the so-called multiple particle model (generalization of the SP model). Meyers et al. [33], combining the impedance of a spherical electrode particle with an electrolyte impedance, obtained an analytical expression for the impedance of a porous electrode under the assumption about the ohmic character of electrolyte impedance. In [34], the authors proposed the impedance model describing diffusion within the solid electrode particles, charge transfer, charging of the double layer, formation of a solid-electrolyte interface, and the concentration gradients inside the electrolyte. Guo et al. [31] studied the energy balance within the SP model and described the thermal behavior of LIB in the process of galvanostatic charge-discharge modes. Note that existing versions of the P2D and SP models do not take the anomalous diffusive propagation of ions in porous electrodes, the solid-electrolyte interface, and amorphous phases into account. Variations of LIB discharge curves calculated within the SP model under normal diffusion conditions are demonstrated in Figure 2. The parameters and formula of the model are given below. 

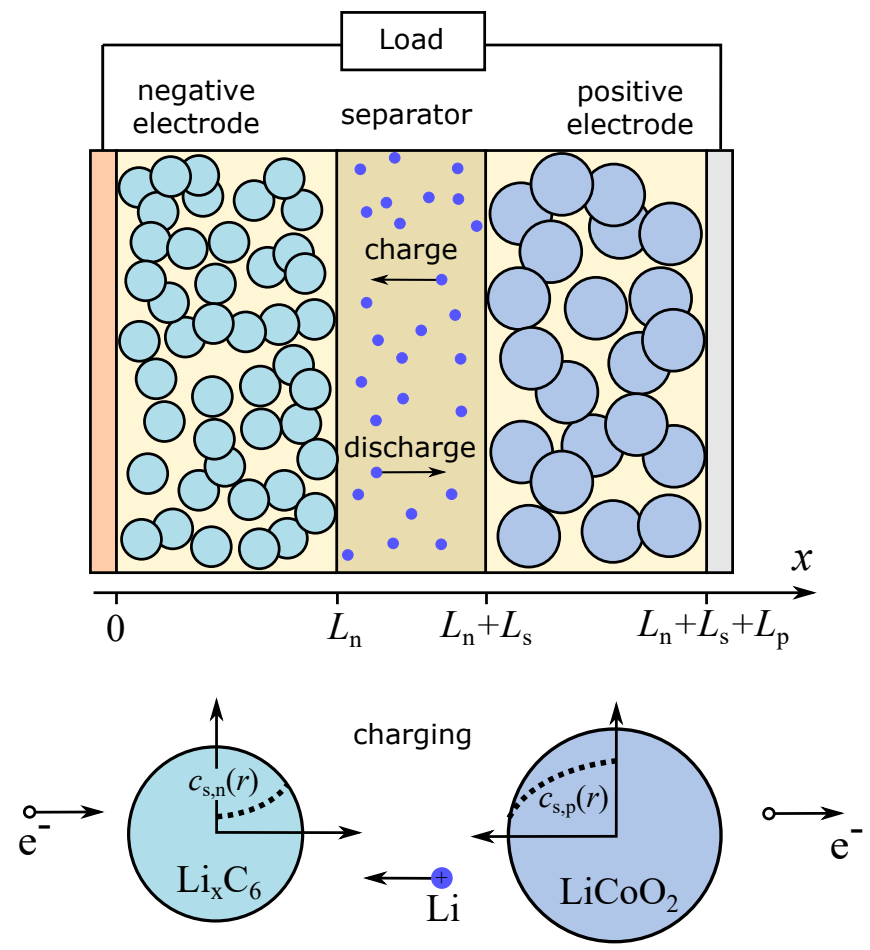

Figure 1. The pseudo-2D model of the Li-ion battery and the single-particle model.
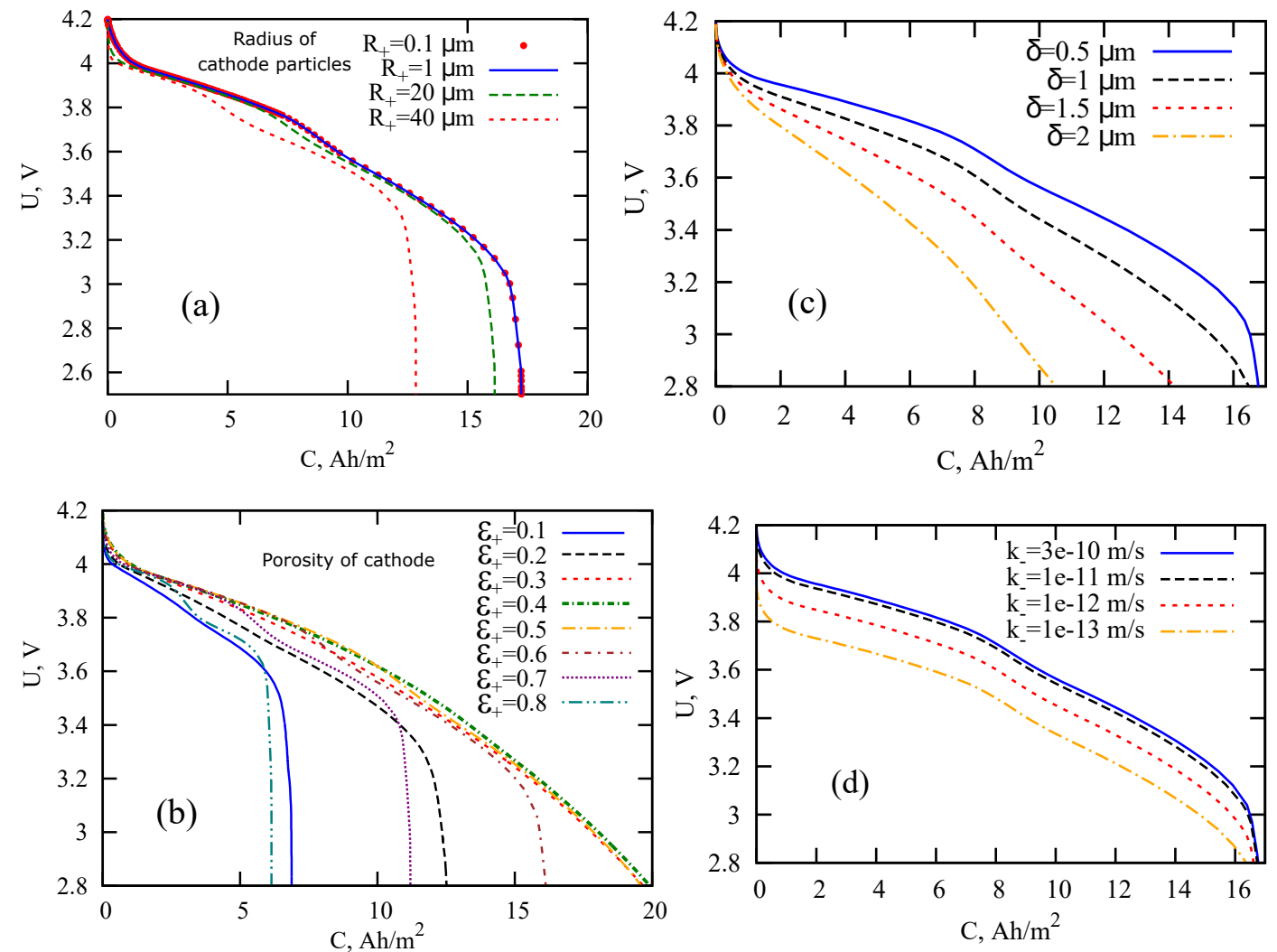

Figure 2. LIB discharge curves calculated within the SP model under normal diffusion conditions for the variation of different parameters: cathode particle radius (a), cathode porosity (b), solid-electrolyte interface (SEI) thickness (c), and electrochemical reaction rate in the anode (d). Other parameters are listed in Table 1, subdiffusion indexes are taken equal to one. 
We consider the general case of subdiffusive motion of lithium in spherical electrode particles and electrolyte. The fractional-order equation of isotropic subdiffusion in spherical coordinates has the form:

$$
\frac{\partial c_{s, j}(r, t)}{\partial t}=K_{s, j} D_{t}^{1-\alpha_{j}} \frac{1}{r^{2}} \frac{\partial}{\partial r}\left(r^{2} \frac{\partial c_{s, j}(r, t)}{\partial r}\right), \quad 0<\alpha_{j} \leq 1,
$$

where $j$ indicates one of the electrodes ( $j=p$ for the cathode and $j=n$ for the anode), $c_{s, j}$ is the lithium concentration inside the particle, $K_{s, j}$ denotes subdiffusion coefficients, $r \in\left[0, r_{j}\right]$ is the radial coordinate, and $r_{j}$ is the radius of spherical particle. The orders $\alpha_{j} \in(0,1]$ of fractional derivatives are equal to the dispersion parameters of ion subdiffusion in solid electrode particles.

The process described by equation (3) implies zero gradients of concentration inside electrodes in the past. It means that the process starts after long relaxation when the distribution of lithium in the electrode particles became uniform, $c_{s, j}(t<0)=c_{s, j}^{0}$. The boundary conditions have the form:

$$
{ }_{0} D_{t}^{1-\alpha_{j}}\left(K_{s, j} \frac{\partial c_{s, j}(r, t)}{\partial r}\right)_{r=0}=0, \quad{ }_{0} D_{t}^{1-\alpha_{j}}\left(K_{s, j} \frac{\partial c_{s, j}(r, t)}{\partial r}\right)_{r=R_{j}}=-J_{j}^{L i}
$$

The first condition implies the absence of current in the particle center, and the second one assumes that the current through the electrode surface is equally distributed between the electrode particles. The molar current density is determined by the expression $J_{j}^{L i}=I / F A_{j}$, where $A_{j}$ is the area of the electrode surface, $F$ is the Faraday constant, and $I$ is the total current flowing through LIB. To write these conditions, the fractional generalization [35] of Fick's law was used.

The current density of ions $J_{j}^{L i}$ is related to overvoltage by the standard Butler-Volmer relation (see, e.g., [36,37]),

$$
J_{j}^{L i}=J_{0, j}\left(1-x_{j}^{\text {surf }}\right)^{0.5}\left(x_{j}^{\text {surf }}\right)^{0.5}\left\{\exp \left[\frac{0.5 F}{R T} \eta_{j}(t)\right]-\exp \left[-\frac{0.5 F}{R T} \eta_{j}(t)\right]\right\},
$$

where the factor $J_{0, j}$ is defined by the formula $J_{0, j}=k_{j} c_{s, j}^{\max } c_{e}^{0.5}$, parameter $k_{j}$ is a reaction rate constant, the transfer coefficients of cathode and anode charge are chosen to be equal to $0.5, R$ and $T$ are the gas constant and temperature, and $c_{s, j}^{\text {surf }}=c_{s, j}\left(r_{j}, t\right)$ is the lithium concentration near the particle surface. Fraction $x_{j}$ determines the state of charge:

$$
x_{j}=\frac{c_{s, j}}{c_{s, j}^{\max }}, \quad x_{j}^{\text {ini }}=\frac{c_{s, j}^{\text {ini }}}{c_{s, j}^{\max }}, \quad x_{j}^{\text {surf }}=\frac{c_{s, j}^{\text {surf }}}{c_{s, j}^{\max }} .
$$

From the Butler-Volmer equation, overvoltage can be expressed as follows [32]:

$$
\eta_{j}(t)=\frac{2 R T}{F} \ln \left[m_{j}(t)+\sqrt{m_{j}^{2}(t)+1}\right], \quad m_{j}(t)=\frac{J_{j}^{L i}}{2 J_{0, j}\left(1-x_{j}^{\text {surf }}\right)^{0.5}\left(x_{j}^{\text {surf }}\right)^{0.5}} .
$$

LIB voltage is equal to the sum of the differences between overvoltages $\eta_{j}$, open circuit voltages $U_{j}$ of electrodes, and voltage drop $\Phi_{e, j}$ in the electrolyte:

$$
V(t)=\eta_{p}\left(x_{p}^{\text {surf }}\right)-\eta_{n}\left(x_{n}^{\text {surf }}\right)+U_{p}\left(x_{p}^{\text {surf }}\right)-U_{n}\left(x_{n}^{\text {surf }}\right)+\left.\Phi_{e, p}(t)\right|_{x=L}-\left.\Phi_{e, n}(t)\right|_{x=0} .
$$

To generalize the results for the case of subdiffusive motion, we use the relation between fundamental solutions of normal and subdiffusion equations. This relationship can be represented in the form [11]:

$$
c_{\alpha}(x, t)=\int_{0}^{\infty} c_{\mathrm{nd}}\left(x, K_{\alpha} t^{\alpha} \tau^{-\alpha}\right) g^{(\alpha)}(\tau) d \tau,
$$


where $c_{\text {nd }}$ is the solution corresponding to the normal diffusion equation with a unit diffusion coefficient, $g_{\alpha}(t)$ is the one-sided stable Lévy density, and $K_{\alpha}$ is the subdiffusion coefficient. The relation can be derived from the representation of the subdiffusion process in terms of a subordinated Brownian motion directed by a fractional Poisson process [23].

Using the solution obtained within the standard SP model and presented, for example, in [31],

$$
x_{j}^{\text {surf }}=x_{j}^{\text {ini }}-\mu_{j}\left[3 \frac{K_{s, j}}{R_{j}^{2}} t+\frac{1}{5}-\sum_{k=1}^{\infty} \frac{2}{\lambda_{k}^{2}} \exp \left(-\frac{\lambda_{k}^{2} K_{s, j}}{R_{j}^{2}} t\right)\right],
$$

where parameter $\mu_{j}=J_{j}^{L i} R_{j} /\left(c_{s, j}^{\max } K_{s, j}\right)$, and $\lambda_{k}$ are the roots of algebraic equation $\sin \lambda_{k}=\lambda_{k} \cos \lambda_{k}$ we arrive at the expression for the subdiffusive case with characteristic exponent $\alpha_{j}$ :

$$
x_{j}^{\text {surf }}=x_{j}^{\text {ini }}-\mu_{j}\left[3 \frac{K_{s, j}}{R_{j}^{2}} \frac{t^{\alpha_{j}}}{\Gamma\left(1+\alpha_{j}\right)}+\frac{1}{5}-\sum_{k=1}^{\infty} \frac{2}{\lambda_{k}^{2}} E_{\alpha_{j}}\left(-\frac{\lambda_{k}^{2} K_{s, j}}{R_{j}^{2}} t^{\alpha_{j}}\right)\right],
$$

which includes the Mittag-Leffler function playing a central role in the fractional differential calculus,

$$
E_{\alpha}(z)=\sum_{n=0}^{\infty} \frac{z^{n}}{\Gamma(1+\alpha n)}
$$

The Mittag-Leffler function in our solution arises as a result of applying Relation (5) to Solution (6) involving the integral representation:

$$
E_{\alpha}\left(-t^{\alpha}\right)=\int_{0}^{\infty} \mathrm{e}^{-t^{\alpha} / \tau^{\alpha}} g^{(\alpha)}(\tau) d \tau
$$

The gamma function appears from the expression for the negative moment of the one-sided stable random variable:

$$
\int_{0}^{\infty} \tau^{-\alpha} g^{(\alpha)}(\tau) d \tau=\frac{1}{\Gamma(1+\alpha)}
$$

By analogy with the normal-diffusion case [38], if we limit ourselves to a parabolic profile, the concentration near the surface is approximately equal to:

$$
x_{j}^{\text {surf }} \approx x_{j}^{\text {ini }}-\mu_{j}\left[3 \frac{K_{s, j}}{R_{j}^{2}} \frac{t^{\alpha_{j}}}{\Gamma\left(1+\alpha_{j}\right)}+\frac{1}{5}-\frac{2}{5} E_{\alpha_{j}}\left(-35 \frac{K_{s, j}}{R_{j}^{2}} t^{\alpha_{j}}\right)\right] .
$$

For given fractions $x_{j}^{\text {surf }}$, we determine overvoltage and open-circuit voltage and then the LIB voltage according to (4). Expressions for the open-circuit voltage $U_{j}$ as a function of the state of charge can be obtained by fitting experimental curves. Expressions for the functions $U_{j}\left(x_{j}\right)$ for $\mathrm{Li}_{x} \mathrm{CoO}_{2}$ and graphite with spherical particles under normal conditions are given, for example, in Appendix A (Formulas (A1) and (A2)) of [31].

In the simplest versions of the SP model, the potential distribution inside the electrolyte is not considered. To model a voltage drop over the electrolyte, the ohmic resistance $R_{\text {cell }}$ is chosen:

$$
\left.\Phi_{e, p}(t)\right|_{x=L}-\left.\Phi_{e, n}(t)\right|_{x=0} \approx I R_{\text {cell }}
$$

where $I$ is the current density in LIB. The work in [31] presented the approximation of $R_{\text {cell }}$ as an empirical function of the ambient temperature and current in LIB. In [39], resistance $R_{\text {cell }}$ was modeled as a function of the ionic conductivity of the electrolyte and the thicknesses of the electrodes. At high charge or discharge rates, the potential distribution inside the electrolyte cannot be neglected [40]. 
To take the distribution of ion concentration across the thickness of the electrodes into account, it is necessary to solve the transport equations in the electrolyte. Under the assumption about ion subdiffusion in the $x$ direction due to the percolative nature of diffusion, we write:

$$
\frac{\partial c_{e, j}}{\partial t}=D_{t}^{1-\beta_{j}}\left[\frac{K_{e, j}^{\mathrm{eff}}}{\varepsilon_{e, j}} \frac{\partial^{2} c_{e, j}}{\partial x^{2}}+\left(1-t_{+}\right) \frac{I}{\varepsilon_{e, j} F L_{j}}\right]
$$

We assume normal diffusion in the separator,

$$
\frac{\partial c_{e, s}}{\partial t}=\frac{K_{e, s}^{\mathrm{eff}}}{\varepsilon_{e, s}} \frac{\partial^{2} c_{e, s}}{\partial x^{2}}
$$

Here, $K_{e, j}^{\text {eff }}=K_{e} \varepsilon_{j}^{b r u g}$ are effective subdiffusion coefficients for ions in electrolyte, $\varepsilon_{j}$ are the porosities of the anode, separator, and cathode, and brug denotes the Bruggeman coefficient.

The boundary conditions at the separator-electrode interfaces correspond to continuity of ion concentration and current. At the cell boundaries $x=0$ and $x=L$, the "reflecting" boundary conditions are applied [32].

Solutions of these equations can be found in the form of polynomials. The orders of these polynomials for electrodes and a separator may vary. Often, the distribution of electrolyte concentration is approximated by a parabolic profile in electrodes and a linear profile in a separator. We use Formulas (14)-(16) from [32] and modify them using the above procedure using Expressions (5), (8), and (9):

$$
\begin{gathered}
c_{e, n}(x, t)=\left(a_{1} x^{2}+a_{2}\right)\left[1-E_{\beta_{n}}\left(-b_{n} t^{\beta_{n}}\right)\right]+c_{0}, \\
c_{e, s}(x, t)=\left(a_{3} x^{2}+a_{4}\right)\left(1-e^{-b_{s} t}\right)+c_{0} \\
c_{e, p}(x, t)=\left(a_{5}(L-x)^{2}+a_{6}\right)\left[1-E_{\beta_{p}}\left(-b_{p} t^{\beta_{p}}\right)\right]+c_{0} .
\end{gathered}
$$

The expressions for the coefficients $a_{i}$ and $b_{i}$ are given in [32] and depend on the thickness and porosity of the cathode, anode, and separator and the ion diffusion coefficient in the electrolyte. Due to the cumbersome nature of these expressions, they are not given here. According to the work [32], we calculate $\left.\Phi_{e, p}(t)\right|_{x=L}-\left.\Phi_{e, n}(t)\right|_{x=0}$ :

$$
\left.\Phi_{2, p}(t)\right|_{x=L}-\left.\Phi_{2, n}(t)\right|_{x=0}=\left(1-t_{+}\right) \frac{2 R T}{F} \ln \frac{c_{e, p}(L, t)}{c_{e, n}(0, t)}-\frac{i_{a p p}}{2}\left(\frac{L_{n}}{\kappa_{n}^{\text {eff }}}+\frac{2 L_{S}}{\kappa_{S}^{\text {eff }}}+\frac{L_{p}}{\kappa_{p}^{\text {eff }}}\right)
$$

Here, $\kappa_{i}^{\text {eff }}=\kappa_{i} \varepsilon_{i}^{\text {brug }}, \kappa_{i}$ is electrolyte conductivity in the cathode, anode, and separator.

After substituting Solution (10) into the expression for the voltage (4), it is possible to calculate the galvanostatic charge-discharge curves in the subdiffusion version of the SP model. Figures 3 and 4 present the calculation results for different values of the subdiffusion parameters. The basic model parameters are listed in Table 1 . The parameters were taken from [16,41] for LIB with the $\mathrm{LiMn}_{2} \mathrm{O}_{4}$ spinel cathode, graphite-based anode, and electrolyte $\mathrm{LiPF}_{6}$ in 1:2 ethylene carbonate and dimethyl carbonate (EC:DMC). The discharge current density was chosen equal to $-15 \mathrm{~A} / \mathrm{m}^{2}$. Decreasing the value of the subdiffusion indices $\alpha_{p}$ and/or $\alpha_{n}$ led to a faster LIB discharge. The new aspect of the degradation of battery performance may be associated, among other factors, with a change in the type of diffusion in LIB components. To verify this statement, let us consider the effect of anomalous diffusion on the LIB impedance and discuss the possibility of determining the anomalous diffusion parameters in individual components using electrochemical impedance spectroscopy. 


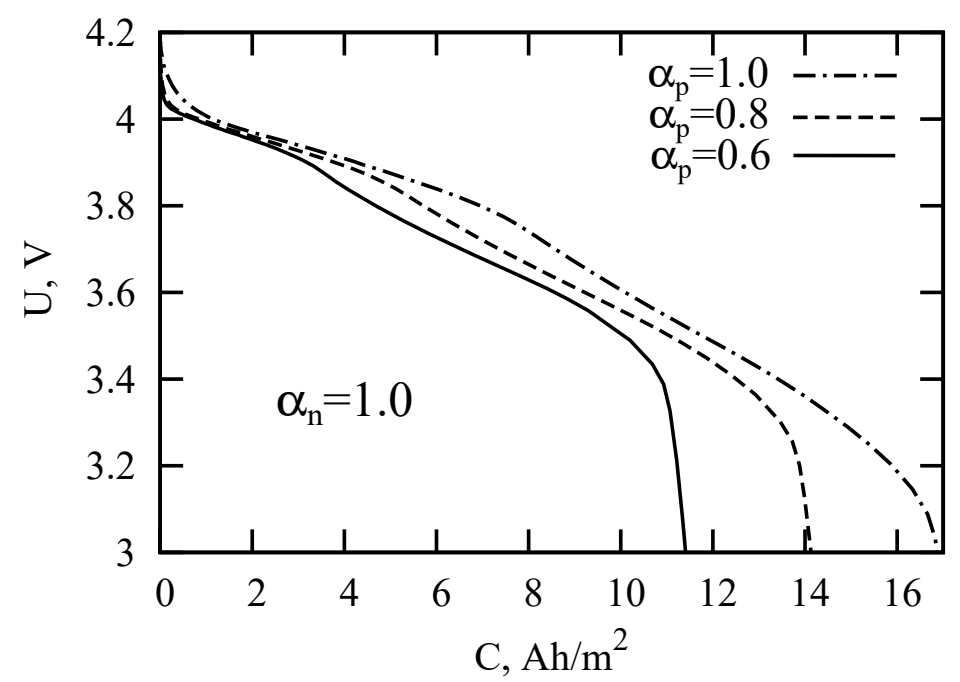

Figure 3. LIB discharge curves for the parameters specified in Table 1 and different values of the subdiffusion index in a cathode.

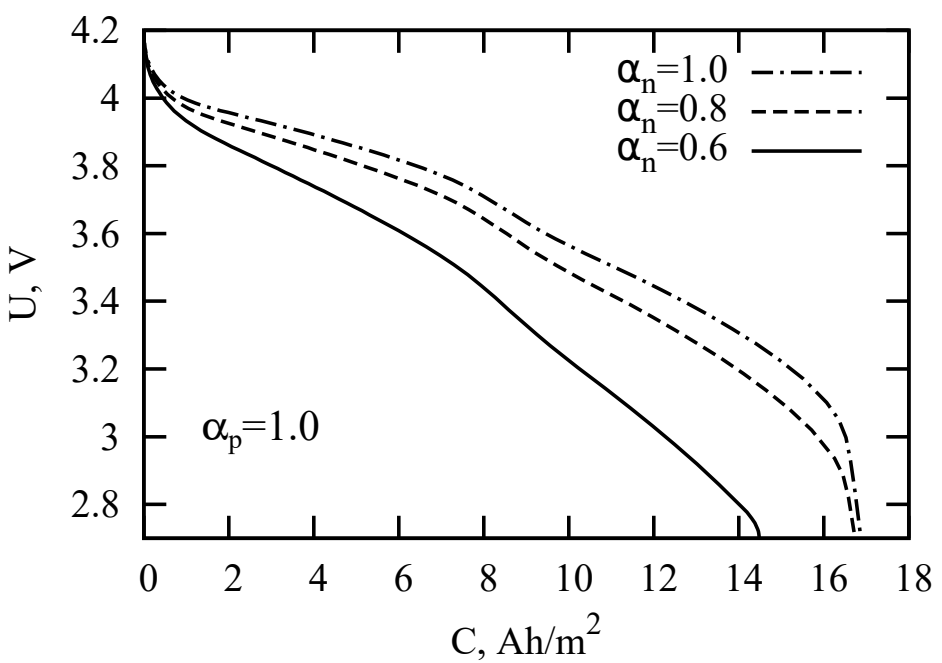

Figure 4. LIB discharge curves for the parameters specified in Table 1 and different values of the subdiffusion index in an anode.

Table 1. SP model parameters.

\begin{tabular}{lccccc}
\hline Parameter & Notation & Units & Cathode & Anode & Separator \\
\hline Thickness & $L_{j}$ & $\mu \mathrm{m}$ & 100 & 100 & 50 \\
Particle radius & $r_{j}$ & $\mu \mathrm{m}$ & 10 & 5 & - \\
Saturation concentration & $c_{s, j}^{\max }$ & $\mathrm{mol} / \mathrm{m}^{3}$ & 20,000 & 25,000 & - \\
Initial concentration & $c_{s, j}^{\mathrm{ini}}$ & $\mathrm{mol} / \mathrm{m}^{3}$ & 4000 & 15,000 & - \\
Subdiffusion coefficient 1 & $K_{s, j}$ & $\mathrm{~m}^{2} / \mathrm{s}^{\alpha_{j}}$ & $10^{-13}$ & $5 \times 10^{-14}$ & - \\
Reaction rate constant & $k_{j}$ & $\mathrm{~m} / \mathrm{s}$ & $5 \times 10^{-10}$ & $4.5 \times 10^{-10}$ & - \\
Subdiffusion coefficient 2 & $K_{e, j}$ & $\mathrm{~m}^{2} / \mathrm{c}^{\beta_{j}}$ & $5 \times 10^{-11}$ & $5 \times 10^{-11}$ & $5 \times 10^{-11}$ \\
Porosity & $\varepsilon_{j}$ & - & 0.6 & 0.45 & 0.5 \\
Bruggeman coefficient & $\mathrm{brug}$ & - & 2.5 & 2.5 & 2.5 \\
\hline
\end{tabular}

\section{Cell Impedance in the Subdiffusion Model}

One of the electrochemical models describing LIB impedance spectra was proposed by Abraham, Kawauchi, and Dees (AKD) in [42]. The model exploits the one-dimensional reaction-diffusion equations, and diffusion is assumed to be normal. The general methodology of the AKD model follows 
the Newmann approach [43], where volume-averaged transport equations for concentrated solutions are used to describe ion transfer in porous separator and composite electrodes. Using expressions for impedances derived by Abraham et al. [42], one could follow changes in impedance spectra due to variations of physical parameters such as electronic conductivities of electrodes, lithium diffusion coefficients, porosities, etc. The values of the basic parameters used in this work for the AKD model are listed in Table 2. The parameters were taken from [16] for LIB with the $\mathrm{LiCoO}_{2}$ cathode, graphite-based anode, and electrolyte LiPF6 in 1:1 ethylene carbonate and diethyl carbonate (EC:DEC). The values of the subdiffusion coefficients were taken close to their normal diffusion analogues. The effects of parameter variations on Nyquist plots are presented in Figure 5. Noteworthy is that the apparent slope of the low frequency (quasi-rectilinear) part at the Nyquist diagrams changes with variations of the lithium diffusion coefficient and porosity, which can be interpreted as a sign of anomalous diffusion [6]. That Nyquist plots at low frequencies have slopes different from $45^{\circ}$ could be explained by the joint contribution of ion diffusion in different components (cathode and anode particles, separator, electrolyte, SEI) characterized by different coefficients. As a result, we observed transitions between different normal diffusive processes prevalent in different frequency ranges.

Table 2. Basic parameters of the Abraham, Kawauchi, and Dees (AKD)-model.

\begin{tabular}{lccccc}
\hline Parameter & Notation & Units & Cathode & Anode & Separator \\
\hline Thickness & $L_{j}$ & $\mu \mathrm{m}$ & 50 & 50 & 30 \\
Temperature & $T$ & $\mathrm{~K}$ & 300 & 300 & 300 \\
Electronic conductor fraction & $\varepsilon_{e c, j}$ & - & 0.2 & 0.15 & - \\
Porosity & $\varepsilon_{j}$ & - & 0.3 & 0.3 & 0.4 \\
Active material fraction & $\varepsilon_{a c t, j}$ & - & 0.3 & 0.3 & 0.3 \\
Bruggeman coefficient & $B_{j}$ & - & 3 & 3 & 3 \\
Diffusion coefficient in the solid phase & $D_{j}$ & $\mathrm{~m}^{2} / \mathrm{s}$ & $5 \times 10^{-13}$ & $7 \times 10^{-15}$ & - \\
Diffusion coefficient in the electrolyte & $D_{e, j}$ & $\mathrm{~m}^{2} / \mathrm{c}$ & $5 \times 10^{-11}$ & $5 \times 10^{-11}$ & $5 \times 10^{-11}$ \\
Electronic conductivity & $\sigma_{j}$ & $\mathrm{~S} / \mathrm{m}$ & 1.13 & 100 & 0 \\
Reference concentration & $C_{j}$ & $\mathrm{~mol}^{3} \mathrm{~m}^{3}$ & $5.6 \times 10^{4}$ & $3.2 \times 10^{4}$ & - \\
Double layer capacitance & $C_{\mathrm{dl}}$ & $\mathrm{F} / \mathrm{m}^{2}$ & 0.25 & 0.25 & - \\
Exchange current density & $i_{j}$ & $\mathrm{~A} / \mathrm{m}^{2}$ & 1 & 1 & - \\
\hline
\end{tabular}

The case of "non-ideal" diffusion behavior, when straight sections on Nyquist diagrams have a slope that differs from $45^{\circ}$, was successfully explained in $[6,44]$ within the framework of the impedance theory of anomalous diffusion [7]. For the sake of fairness, it is worth noting that the low-frequency parts of Nyquist plots observed by [6] were straighter than shown in Figure 5. Therefore, the anomalous diffusion interpretation is a plausible hypothesis. Here, we want to coordinate fractional differential generalization of the SP model with an equivalent circuit model and related impedance spectra.

The Fourier transform of Equation (2) leads to the expression:

$$
(i \omega)^{\alpha} \tilde{c}(x, \omega)=K \Delta \tilde{c}(x, \omega) .
$$

In this case, the modification of the impedance in the case of subdiffusion is not limited to a simple replacement $(i \omega)$ on $(i \omega)^{\alpha}$ : the standard Fick law in the case of anomalous diffusion is not satisfied. For the diffusion Equation (2), the expression for the flux density is the following:

$$
J(x, t)=K_{0} \mathrm{D}_{t}^{1-\alpha} \frac{\partial c(x, t)}{\partial x} .
$$

In particular, the subdiffusion generalization of Warburg's impedance for a semi-infinite medium has the form [7]:

$$
Z(j \omega) \propto(i \omega)^{-(1-\alpha / 2)}
$$


For spherical particles, the Fourier transform of the subdiffusion Equation (3) leads to the differential equation:

$$
(i \omega)^{\alpha_{j}} \tilde{c}_{s, j}(r, \omega)=K_{s, j} \frac{1}{r^{2}} \frac{\partial}{\partial r}\left(r^{2} \frac{\partial \tilde{c}_{s, j}(r, \omega)}{\partial r}\right), \quad 0<\alpha_{j} \leq 1, \quad 0<r<r_{j} .
$$

Using the standard procedure (see for example [32]) from Equation (14) under the relevant boundary conditions and applying the generalized Fick law, one can obtain a generalization of Faraday's reaction impedance:

$$
Z_{s, j}=R_{c t, j}+\frac{R_{s, j}}{r_{j} K_{s, j}^{-1 / 2}(i \omega)^{1-\alpha_{j} / 2} \operatorname{coth}\left\{r_{j} K_{s, j}^{-1 / 2}(i \omega)^{\alpha_{j} / 2}\right\}-1} .
$$

It has the form of an impedance corresponding to a series connections of charge transfer resistance $R_{c t, j}$ and impedance associated with subdiffusion in the solid phase,

$$
R_{c t, j}=\frac{R T}{F J_{0, j}}, \quad R_{s, j}=\frac{r_{j} R T}{F^{2} K_{s, j} c_{s, j}^{\text {surf }}\left(1-k_{j} c_{s, j}^{\text {surf }}\right)} .
$$
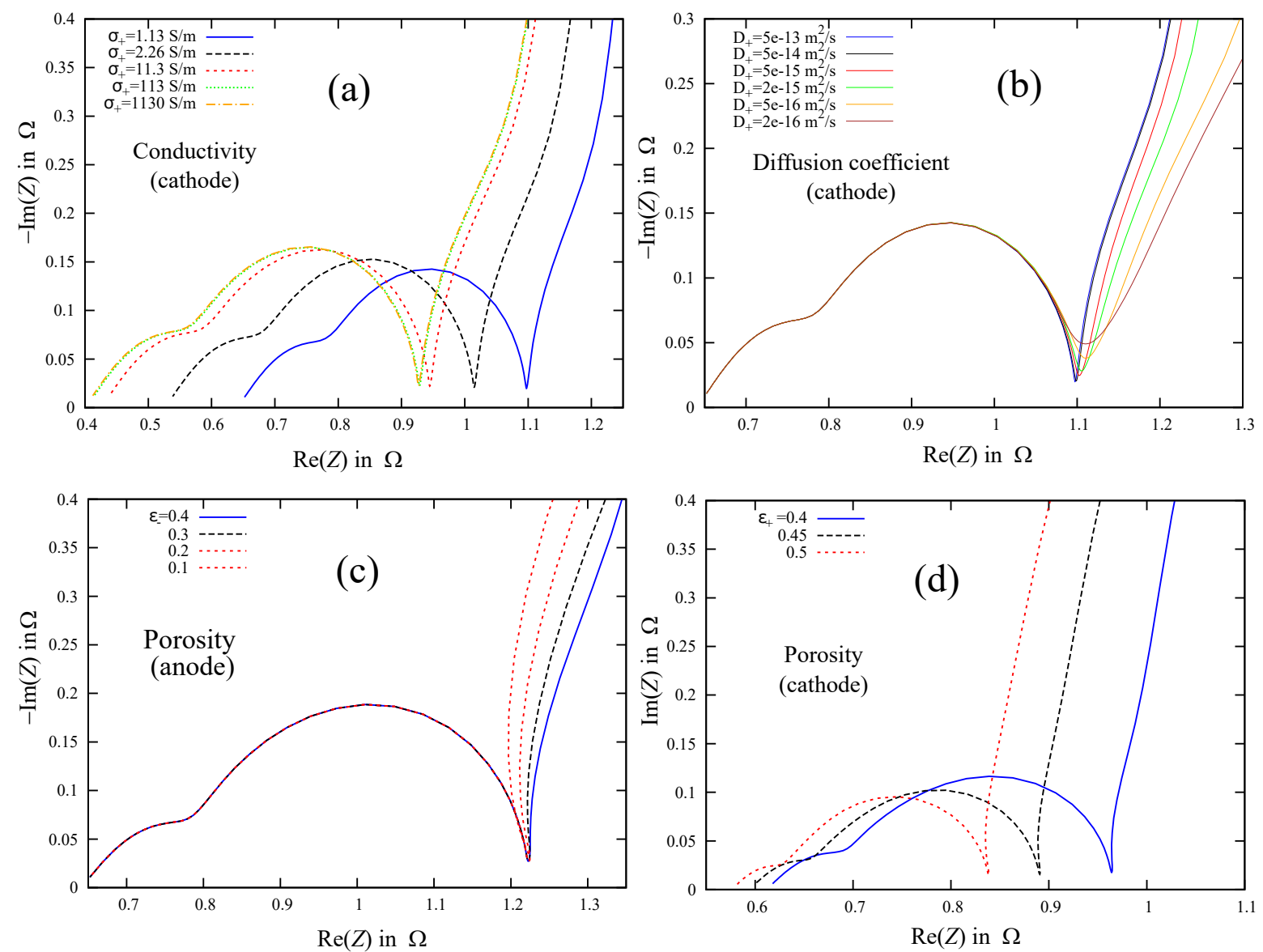

Figure 5. Nyquist diagrams for variations of electrode parameters: electronic conductivity in cathode (a), diffusion coefficient for ions in cathode particles (b), porosity of anode (c), and porosity of cathode (d). 
Assume ion subdiffusion with exponents $\beta_{j}$ in electrolyte filling the $j$ electrode (cathode or anode). The impedance of a double layer formed on the surface of a spherical particle can be found by linearizing the transport equation using the Fourier transform:

$$
(i \omega)^{\beta_{j}} \tilde{c}_{e, j}(r, \omega)=K_{e, j} \frac{1}{r^{2}} \frac{\partial}{\partial r}\left(r^{2} \frac{\partial \tilde{c}_{e, j}(r, \omega)}{\partial r}\right), \quad r>r_{j} .
$$

Solving this equation, in analogy with [45], we arrive at the subdiffusion impedance of a double layer on a spherical particle:

$$
Z_{\mathrm{DL}}=\frac{r_{j} R T F^{-2} K_{e, j}^{-1}}{1+r_{j} K_{e, j}^{-1 / 2}(i \omega)^{1-\beta_{j} / 2}} .
$$

The latter expression can be considered as an impedance of parallel connection of a constant phase element and a resistor:

$$
\mathrm{Z}_{\mathrm{DL}}^{\mathrm{CPE}}=\frac{R T}{F^{2} K_{e, j}^{1 / 2}}(i \omega)^{-\left(1-\beta_{j} / 2\right)}, \quad R_{\mathrm{DL}}=\frac{r_{j} R T}{F^{2} K_{e, j}}
$$

The impedance of the anode film is found as the subdiffusion impedance of the spherical layer according to the procedure presented in [45], but using the generalized Fick law. The result represents a parallel connection of the film resistance $R_{f}$ and the subdiffusive generalization of Warburg open impedance:

$$
Z_{f}^{\prime}(\omega)=C_{\gamma} K_{f}^{-1 / 2}(j \omega)^{-(1-\gamma / 2)} \tanh \left[\delta_{f} K_{f}^{-1 / 2}(j \omega)^{\gamma / 2}\right],
$$

where $\gamma$ and $K_{f}$ are the dispersion parameter and subdiffusion coefficient for the anode film. Here, however, it should be noted that SEI thickness is usually several nanometers, and the diffusion limit may not be fulfilled on such small scales, while the validity of Expression (16) remains in question.

Assuming independent parallel flow of the charge current due to an electric double layer formation and intercalation current on the active material surface according to [46], after arranging the impedances of the electrodes, separator, and current collector, we arrive at the equivalent circuit shown in Figure 6. This circuit summarizes the popular LIB models (see, for example, [3,32]). Under certain simplifications, the equivalent circuit (EC) presented in Figure 6 can be reduced to the model considered in [3]. Our scheme is also consistent with the one proposed in [32]. However, Li et al. [32] introduced "by-hand" three CPE, substantiating them with the roughness of electrode particles and the fragmented SEI structure. Without denying the plausibility of these arguments, we only note that in our case, the circuit was fully derived within a methodologically-consistent model assuming subdiffusion in the electrolyte filling porous medium and in electrode particles.

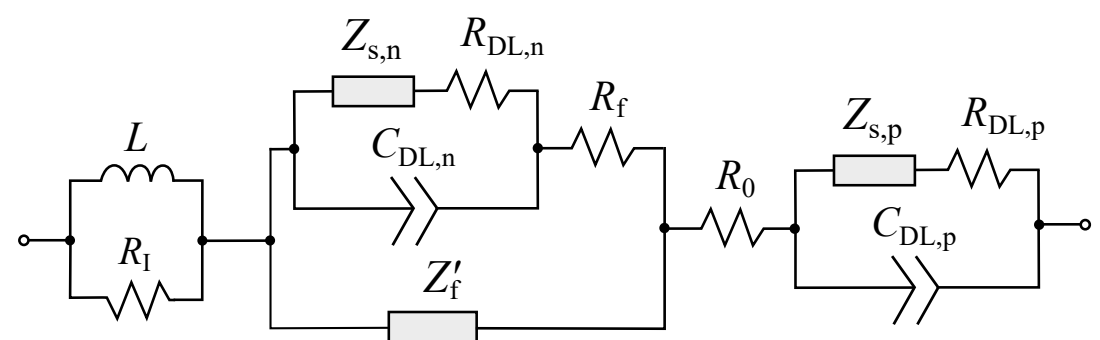

Figure 6. The LIB equivalent circuit model obtained on the basis of the subdiffusion generalization of the electrochemical SP model.

Consider the simplified circuit presented in Figure 7. The Nyquist diagrams are also presented for four pairs of subdiffusion index values $\left(\alpha_{n}, \alpha_{p}\right):(1,1),(1,0.5),(0.5,0.5)$, and $(0.5,1)$. For the other parameters: $L=0.12 \mu \mathrm{H}, R_{I}=0.31 \mathrm{Ohm}, C_{\mathrm{DL}, p}=0.026 \mathrm{~F}, C_{\mathrm{DL}, n}=0.0066 \mathrm{~F}, R_{0}=0.12 \mathrm{Ohm}$, $R_{\mathrm{DL}, p}+R_{c t, p}=0.11 \mathrm{Ohm}, R_{\mathrm{DL}, n}+R_{c t, n}=0.026 \mathrm{Ohm}$. It can be seen from these diagrams that ion 
subdiffusions in the cathode and anode have a joint effect on the slope of the low-frequency section. Thus, the slope of this section cannot uniquely determine the subdiffusion indices $\alpha_{n}$ and $\alpha_{p}$ separately from each other.

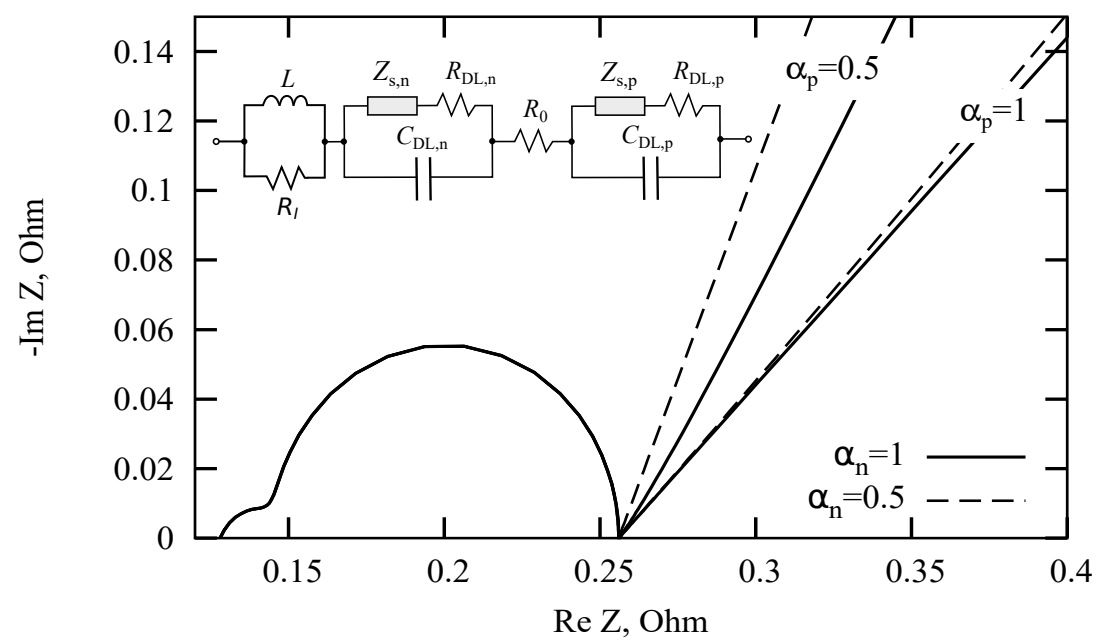

Figure 7. The change in slope of the rectilinear part of the Nyquist diagram with variations in subdiffusion indices.

Adverse reactions and degradation processes can lead to a number of undesirable effects associated with the loss of LIB capacity. Usually, LIB aging occurs due to a variety of processes and reactions that simultaneously occur in different areas of LIB. A decrease in LIB capacity is often associated with processes occurring in a negative graphite electrode, where the formation of SEI film leads to an irreversible loss of cycled lithium, to an increase in internal resistance, and to a decrease in the pore volume available for the electrolyte. In our opinion, another additional factor is possible. It is associated with an increase in the fraction of particles, in which anomalous ion diffusion is predominant. The calculated discharge curves in galvanostatic mode (Figures 3 and 4) indicate the plausibility of the hypothesis. An increase in the role of anomalous diffusion due to the disordering of the crystal lattice or the formation of an amorphous film can be estimated by introducing parallel-connected elements (see Figure 8, inset). Suppose that during the cycling, the $\varepsilon$ fraction of particles characterized by anomalous diffusion of lithium in the solid phase increases. Let us choose the cathode as an example. The Nyquist diagrams for different values of $\varepsilon$ are shown in Figure 8 . The model parameters are the same as for Figure 7 with $R_{\mathrm{DL}, p}^{1}+R_{c t, n}^{1}=0.024 \mathrm{Ohm}$. The diffusion coefficients are taken from Table 1 . Elements $Z_{s, p}^{\alpha_{p}}$ and $R_{\mathrm{DL}, p}$ are multiplied by $\varepsilon^{-1}$ and the elements $Z_{s, p}^{1}$ and $R_{\mathrm{DL}, p}^{1}$ by $(1-\varepsilon)^{-1}$. It can be seen from these diagrams that the slope of the rectilinear segment at low frequencies does not always unambiguously determine the subdiffusion index $\alpha$ and can be both larger and smaller than the slope corresponding to normal diffusion. 


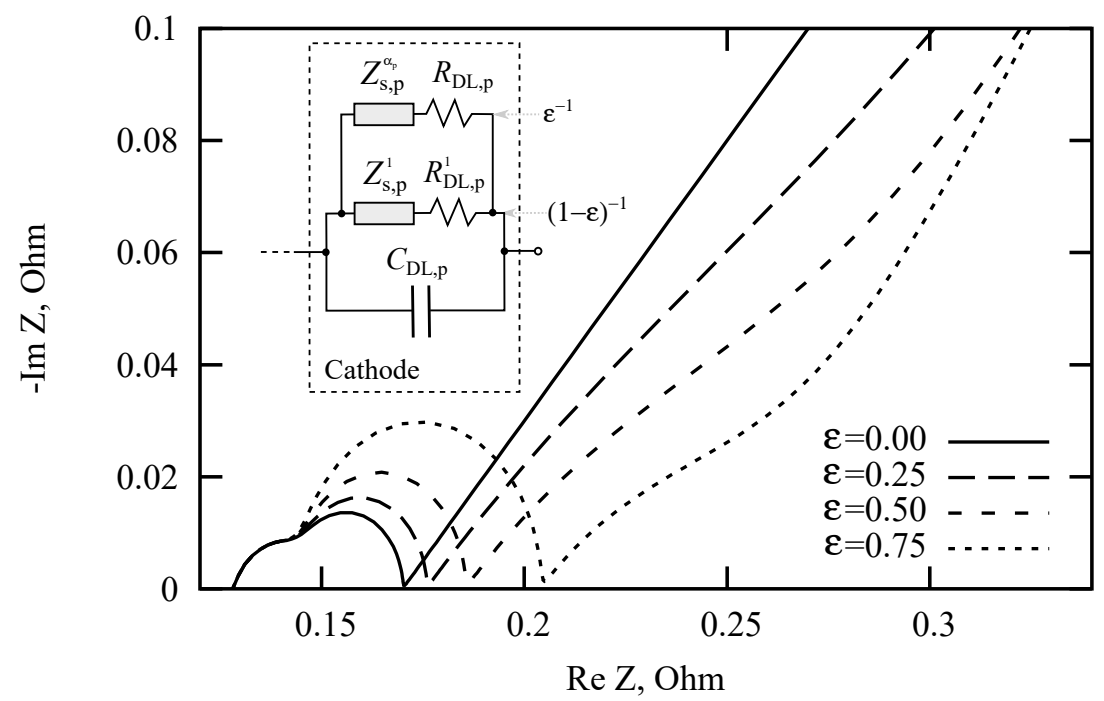

Figure 8. Nyquist diagrams for different values of the $\varepsilon$ fraction of cathode particles characterized by ion subdiffusive transport. The $1-\varepsilon$ fraction of particles is characterized by normal diffusion.

\section{Conclusions}

In LIB, there are many factors that can sustain the anomalous-diffusive behavior of ions, such as the percolative structure of porous electrodes, an amorphous solid-electrolyte interface, grain boundaries in electrodes and the electrolyte, etc. In our work, the influence of subdiffusion on the galvanostatic discharge and LIB impedance was evaluated within the generalization of the single-particle model. This generalization was based on the fractional differential equations of ion diffusion in electrode particles, SEI, and electrolyte. The distribution of lithium ions in electrolyte and electrode particles was expressed via the Mittag-Leffler function and the Lévy stable density. It was shown that subdiffusion in the solid phase of electrodes and in electrolyte deteriorated the performance of LIB. The calculated discharge curves indicated an acceleration of voltage drop at lower values of subdiffusion index. Within the framework of the new model, an equivalent circuit was derived. It generalized the popular models of LIB. The slope of the rectilinear part of the Nyquist diagram at low frequencies did not always unambiguously determine the subdiffusion index $\alpha$ and could be either larger or smaller than the slope corresponding to normal diffusion. It was shown that the degradation of battery properties can be associated, among other factors, with a change in the type of diffusion in LIB components.

Author Contributions: Methodology R.T.S.; validation, E.P.K. and V.V.S.; resources, V.V.S. and A.A.P; writing, original draft preparation, R.T.S.; writing, review and editing, R.T.S., E.P.K., and V.V.S.; supervision, R.T.S.; project administration, V.V.S. and A.A.P.; funding acquisition, V.V.S., R.T.S., and A.A.P.

Funding: R.T.S. thanks the Russian Foundation for Basic Research (Grants 18-51-53018 and 18-42-732002) and the Ministry of Science and Higher Education of the Russian Federation (Project 3.2111.2017/4.6) for financial support.

Acknowledgments: We are grateful to our colleagues Sergey Novikov, Andrey Somov, Pavel L'vov, Ekaterina Morozova, Pavel Kapustin, and Dmitry Vostretzov from the Ulyanovsk State University for the discussion of particular problems related to this work.

Conflicts of Interest: The authors declare no conflict of interest.

\section{Abbreviations}

The following abbreviations are used in this manuscript:

$\begin{array}{ll}\text { LIB } & \text { lithium-ion battery } \\ \text { GB } & \text { grain boundary } \\ \text { SP } & \text { single particle } \\ \text { EDL } & \text { electric double layer } \\ \text { EC } & \text { equivalent circuit } \\ \text { CPE } & \text { constant phase element }\end{array}$




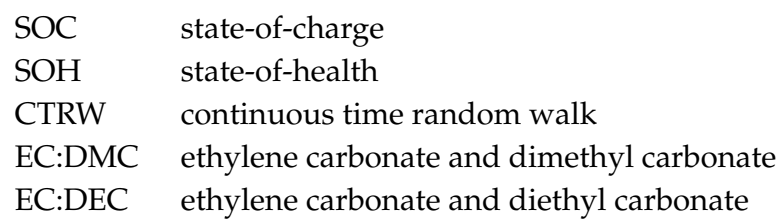

\section{References}

1. Thomas, K.E.; Newman, J.; Darling, R.M. Mathematical modeling of lithium batteries. In Advances in Lithium-Ion Batteries; Springer: Boston, MA, USA, 2002; pp. 345-392.

2. Hariharan, K.S.; Tagade, P.; Ramachandran, S. Mathematical Modeling of Lithium Batteries: From Electrochemical Models to State Estimator Algorithms; Springer: New York, NY, USA, 2017.

3. Osaka, T.; Momma, T.; Mukoyama, D.; Nara, H. Proposal of novel equivalent circuit for electrochemical impedance analysis of commercially available lithium ion battery. J. Power Sources 2012, 205, 483-486. [CrossRef]

4. Freeborn, T.J.; Maundy, B.; Elwakil, A.S. Fractional-order models of supercapacitors, batteries and fuel cells: A survey. Mater. Renew. Sustain. Energy 2015, 4, 1-7. [CrossRef]

5. Sabatier, J. Fractional order models for electrochemical devices. Fract. Dyn. 2015, 141, 141-160.

6. Erol, S.; Orazem, M.E. The influence of anomalous diffusion on the impedance response of $\mathrm{LiCoO}_{2} \mid \mathrm{C}$ batteries. J. Power Sources 2015, 293, 57-64. [CrossRef]

7. Bisquert, J.; Compte, A. Theory of the electrochemical impedance of anomalous diffusion. J. Electroanal. Chem. 2011, 499, 112-120. [CrossRef]

8. Rollet, A.-L.; Porion, P.; Vaultier, M.; Billard, I.; Deschamps, M.; Bessada, C.; Jouvensal, L. Anomalous diffusion of water in [BMIM][TFSI] room-temperature ionic liquid. J. Phys. Chem. 2007, 111, 11888-11891. [CrossRef] [PubMed]

9. Weber, H.W.; Kimmich, R. Anomalous segment diffusion in polymers and NMR relaxation spectroscopy. Macromolecules 1993, 26, 2597-2606. [CrossRef]

10. Hayamizu, K.; Aihara, Y.; Price, W.S. Correlating the NMR self-diffusion and relaxation measurements with ionic conductivity in polymer electrolytes composed of cross-linked poly (ethylene oxide-propylene oxide) doped with $\mathrm{LiN}\left(\mathrm{SO}_{2} \mathrm{CF}_{3}\right)_{2}$. J. Chem. Phys. 2000, 113, 4785-4793. [CrossRef]

11. Uchaikin, V.V. Self-similar anomalous diffusion and Lévy-stable laws. Physics-Uspekhi 2003, 46, 821. [CrossRef]

12. Sun, H.; Chen, W.; Li, C.; Chen, Y. Fractional differential models for anomalous diffusion. Phys. A Stat. Mech. Its Appl. 2010, 389, 2719-2724. [CrossRef]

13. Metzler, R.; Klafter, J. The random walk's guide to anomalous diffusion: A fractional dynamics approach. Phys. Rep. 2000, 339, 1-77. [CrossRef]

14. Sabatier, J.; Merveillaut, M.; Francisco, J.M.; Guillemard, F.; Porcelatto, D. Lithium-ion batteries modeling involving fractional differentiation. J. Power Sources 2014, 262, 36-43. [CrossRef]

15. Sabatier, J.; Francisco, J.M.; Guillemard, F.; Lavigne, L.; Moze, M.; Merveillaut, M. Lithium-ion batteries modeling: A simple fractional differentiation based model and its associated parameters estimation method. Signal Process. 2015, 107, 290-301. [CrossRef]

16. Park, M.; Zhang, X.; Chung, M.; Less, G.B.; Sastry, A.M. A review of conduction phenomena in Li-ion batteries. J. Power Sources 2010, 195, 7904-7929. [CrossRef]

17. Sibatov, R.; Svetukhin, V. Grain boundary diffusion in terms of the tempered fractional calculus. Phys. Lett. A 2017, 381, 2021-2027. [CrossRef]

18. Sibatov, R.T. Anomalous Grain Boundary Diffusion: Fractional Calculus Approach. Adv. Math. Phys. 2019, 2019, 8017363. [CrossRef]

19. Huang, J. Generalization of porous electrode theory for noninteger dimensional space. J. Phys. Chem. C 2017, 122, 557-565. [CrossRef]

20. Chechkin, A.V.; Seno, F.; Metzler, R.; Sokolov, I.M. Brownian yet non-Gaussian diffusion: From superstatistics to subordination of diffusing diffusivities. Phys. Rev. X 2017, 7, 021002. [CrossRef]

21. Sibatov, R.T.; Uchaikin, V.V. Fractional differential approach to dispersive transport in semiconductors. Physics-Uspekhi 2009, 52, 1019. [CrossRef] 
22. Samko, S.G.; Kilbas, A.A.; Marichev, O.I. Fractional Integrals and Derivatives: Theory and Applications; Gordon and Breach Science Publishers: Philadelphia, PA, USA, 1993.

23. Uchaikin, V.V.; Sibatov, R. Fractional kinetics in solids: Anomalous charge transport in semiconductors, dielectrics, and nanosystems. World Sci. 2013._0001. [CrossRef]

24. Weiss, G.H.; Havlin, S. Some properties of a random walk on a comb structure. Phys. A Stat. Mech. Its Appl. 1986, 134, 474-482. [CrossRef]

25. Arkhincheev, V.; Baskin, E. Anomalous diffusion and drift in a comb model of percolation clusters. Sov. Phys. JETP 1991, 73, 161-300.

26. Arkhincheev, V. Random walk on hierarchical comb structures. J. Exp. Theor. Phys. 1999, 88, 710-715. [CrossRef]

27. Lubashevskii, I.; Zemlyanov, A. Continuum description of anomalous diffusion on a comb structure. J. Exp. Theor. Phys. 1998, 87, 700-713. [CrossRef]

28. Doyle, M.; Fuller, T.F.; Newman, J. Modeling of galvanostatic charge and discharge of the lithium/polymer/ insertion cell. J. Electrochem. Soc. 1993, 140, 1526-1533. [CrossRef]

29. Doyle, M. Design and Simulation of Lithium Rechargeable Batteries. Ph.D. Thesis, University of California, Berkeley, CA, USA, 1995.

30. Newman, J.S.; Tobias, C.W. Theoretical analysis of current distribution in porous electrodes. J. Electrochem. Soc. 1962, 109, 1183-1191. [CrossRef]

31. Guo, M.; Sikha, G.; White, R.E. Single-particle model for a lithium-ion cell: Thermal behavior. J. Electrochem. Soc. 2011, 158, A122-A132. [CrossRef]

32. Li, S.E.; Wang, B.; Peng, H.; Hu, X. An electrochemistry-based impedance model for lithium-ion batteries. J. Power Sources 2014, 258, 9-18. [CrossRef]

33. Meyers, J.P.; Doyle, M.; Darling, R.M.; Newman, J. The impedance response of a porous electrode composed of intercalation particles. J. Electrochem. Soc. 2000, 147, 2930-2940. [CrossRef]

34. Sikha, G.; White, R.E. Analytical expression for the impedance response of an insertion electrode cell. J. Electrochem. Soc. 2007, 154, A43-A54. [CrossRef]

35. Paradisi, P.; Cesari, R.; Mainardi, F.; Tampieri, F. The fractional Fick's law for non-local transport processes. Phys. A 2001, 293, 130-142. [CrossRef]

36. Bard, A.J.; Faulkner, L.R.; Leddy, J.; Zoski, C.G. Electrochemical Methods: Fundamentals and Applications; Wiley: New York, NY, USA, 1980; Volume 2.

37. Van Soestbergen, M. Frumkin-Butler-Volmer theory and mass transfer in electrochemical cells. Russ. J. Electrochem. 2012, 48, 570-579. [CrossRef]

38. Subramanian, V.R.; Ritter, J.A.; White, R.E. Approximate solutions for galvanostatic discharge of spherical particles i. constant diffusion coefficient. J. Electrochem. Soc. 2001, 148, E444-E449. [CrossRef]

39. Domenico, D.D.; Stefanopoulou, A.; Fiengo, G. Lithium-ion battery state of charge and critical surface charge estimation using an electrochemical model-based extended kalman filter. J. Dyn. Syst. Meas. Control 2010, 132, 061302. [CrossRef]

40. Li, Y.; Wang, L.; Liao, C.; Wang, L.; Xu, D. Recursive modeling and online identification of lithium-ion batteries for electric vehicle applications. Sci. China Technol. Sci. 2014, 57, 403-413. [CrossRef]

41. Doyle, M.; Newman, J.; Gozdz, A.S.; Schmutz, C.N.; Tarascon, J.M. Comparison of modeling predictions with experimental data from plastic lithium ion cells. J. Electrochem. Soc. 1996, 143, 1890-1903. [CrossRef]

42. Abraham, D.P.; Kawauchi, S.; Dees, D.W. Modeling the impedance versus voltage characteristics of $\mathrm{LiNi}_{0.8} \mathrm{Co}_{0.15} \mathrm{Al}_{0.05} \mathrm{O}_{2}$. Electrochim. Acta 2008, 53, 2121-2129. [CrossRef]

43. Newman, J.; Thomas-Alyea, K.E. Electrochemical Systems; John Wiley \& Sons: Hoboken, NJ, USA, 2004.

44. Huang, J. Diffusion impedance of electroactive materials, electrolytic solutions and porous electrodes: Warburg impedance and beyond. Electrochim. Acta 2018, 281, 170-188. [CrossRef]

45. Jacobsen, T.; West, K. Diffusion impedance in planar, cylindrical and spherical symmetry. Electrochim. Acta 1995, 40, 255-262. [CrossRef]

46. Randles, J.E.B. Kinetics of rapid electrode reactions. Discuss. Faraday Soc. 1947, 1, 11-19. [CrossRef]

(c) 2019 by the authors. Licensee MDPI, Basel, Switzerland. This article is an open access article distributed under the terms and conditions of the Creative Commons Attribution (CC BY) license (http:/ / creativecommons.org/licenses/by/4.0/). 